\title{
COUPLING THE STOKES AND NAVIER-STOKES EQUATIONS WITH TWO SCALAR NONLINEAR PARABOLIC EQUATIONS*
}

\author{
Macarena Gómez Mármol ${ }^{1}$ and Francisco Ortegón Gallego ${ }^{2}$
}

\begin{abstract}
This work deals with a system of nonlinear parabolic equations arising in turbulence modelling. The unknowns are the $N$ components of the velocity field $u$ coupled with two scalar quantities $\theta$ and $\varphi$. The system presents nonlinear turbulent viscosity $A(\theta, \varphi)$ and nonlinear source terms of the form $\theta^{2}|\nabla u|^{2}$ and $\theta \varphi|\nabla u|^{2}$ lying in $L^{1}$. Some existence results are shown in this paper, including $L^{\infty}$-estimates and positivity for both $\theta$ and $\varphi$.

Résumé. Nous étudions un système non-linéaire d'équations du type parabolique provenant de la modélisation de la turbulence. Les inconnues sont les $N$ composantes du champ des vitesses $u$ couplées avec deux grandeurs scalaires $\theta$ et $\varphi$. Ce système présente un terme de diffusion non-linéaire sous forme matricielle $A(\theta, \varphi)$ et les termes sources non-linéaires $\theta^{2}|\nabla u|^{2}$ et $\theta \varphi|\nabla u|^{2}$ appartenant à $L^{1}$. On démontre alors quelques résultats d'existence de solutions, ainsi que des estimations dans $L^{\infty}$ et positivité pour $\theta$ et $\varphi$.
\end{abstract}

AMS Subject Classification. 35K45, 35K60, 35Q35.

Received: December 3, 1996. Revised: November 18, 1997.

\section{INTRODUCTION}

In this paper, we state some existence results of a weak solution to the nonlinear parabolic system

$$
\begin{cases}\frac{\partial u}{\partial t}-\nabla \cdot(A(\theta, \varphi) \nabla u)+\nabla p=f, \quad \nabla \cdot u=0, & \text { in } Q \\ \frac{\partial \theta}{\partial t}-\nabla \cdot(A(\theta, \varphi) \nabla \theta)=1-\theta^{2}|\nabla u|^{2}, & \text { in } Q \\ \frac{\partial \varphi}{\partial t}-\nabla \cdot(A(\theta, \varphi) \nabla \varphi)=-\varphi\left(\theta|\nabla u|^{2}+\frac{1}{\theta+r}\right), & \text { in } Q \\ u(x, 0)=u_{0}(x), \quad \theta(x, 0)=\theta_{0}(x), \quad \varphi(x, 0)=\varphi_{0}(x), & \text { in } \Omega \\ u(x, t)=0, \quad \theta(x, t)=a, \quad \varphi(x, t)=b, & \text { on } \partial \Omega \times(0, T)\end{cases}
$$

where $Q=\Omega \times(0, T), \Omega \subset \mathbb{R}^{N}$ is a bounded domain with Lipschitz boundary $\partial \Omega, N \geq 2, T>0, a$ and $b$ are non-negative constants and $r>0$ is a small parameter. Positivity of both $\theta$ and $\varphi$ is also shown in this work.

System $(S)$ derives from the so-called $k$ - $\epsilon$ turbulence model (see [8]). Here, $u=\left(u_{1}, \ldots, u_{N}\right)^{\prime}$ stands for the mean velocity field (the symbol ' here means vector transposition), $p$ is the mean pressure and $f=\left(f_{1}, \ldots, f_{N}\right)^{\prime}$

Key words and phrases. Nonlinear parabolic systems, Stokes and Navier-Stokes equations.

* Research partially supported by DGICYT grant PB93-1196.

${ }^{1}$ Departamento de Ecuaciones Diferenciales y Análisis Numérico Universidad de Sevilla, Spain. e-mail: ortegon@numer.us.es

${ }^{2}$ Departamento de Matemáticas, Universidad de Cádiz, Spain. e-mail: francisco.ortegon@uca.es 
is a given function describing a distributed force field over $Q$. The magnitudes $\theta$ and $\varphi$ are obtained from both, the mean turbulent kinetic energy, $k$, and the mean turbulent viscous dissipation, $\epsilon$. Indeed, $\theta=k \epsilon^{-1}$, $\varphi=\epsilon^{2} k^{-3}$ and the $\theta$ and $\varphi$ equations are both deduced from those of $k$ and $\epsilon$ (see [6-8] for details).

The resulting viscosity is of the form $A(\theta, \varphi)=\left(\nu_{0}+\frac{1}{\theta \varphi}\right) I, \nu_{0}>0$ being a constant value. In order to avoid a zero denominator we must change this expression in some way; for example, we may take a perturbation of the form $A(\theta, \varphi)=\left(\nu_{0}+\frac{1}{|\theta \varphi|+r}\right) I$. Here we shall consider a general matrix expression for $A(\theta, \varphi)$. Also, we point out that the term $-\frac{\varphi}{\theta}$ appearing in the original modelling of the equation for $\varphi$ has been changed to $-\frac{\varphi}{|\theta|+r}$ for the same reason as above.

System $(S)$ lacks transport terms; we have first considered these equations (without transport terms) in order to establish some existence results, independently on the space dimension. The resolution of the full model $(C)$ is discussed in Section 6 below.

As we are just considering system $(S)$ from the mathematical standpoint, we may assume that the involved physical quantities are dimensionless and that the physical constants which actually appear are taken to be equal to one.

The interest in introducing the $\theta-\varphi$ approach in turbulence modelling is that $(S)$ may be considered as a stabilization of the $k-\epsilon$ model in the sense that we can state some results concerning the existence, regularity, positivity and $L^{\infty}$-estimates of certain solutions of $(S)$.

As one can readily see, system $(S)$ presents some mathematical difficulties, namely

1. nonlinear source terms and nonlinear viscosity;

2. the whole system is coupled through these nonlinear terms;

3. the regularity result for the Stokes (or even the Navier-Stokes equations) yields a velocity field $u \in$ $L^{2}\left([0, T] ; H_{0}^{1}(\Omega)^{N}\right)$ if, for example, $f \in L^{2}\left([0, T] ; H^{-1}(\Omega)^{N}\right)$. So both the $\theta$ and $\varphi$ equations contain nonlinear source terms, namely, $\theta^{2}|\nabla u|^{2}$ and $\theta \varphi|\nabla u|^{2}$, which lie in $L^{1}(Q)$ if $\theta$ and $\varphi$ belong to, say, $L^{\infty}(Q)$.

Some partial results concerning the existence and positivity of solutions $(\theta, \varphi)$ may be found in $[6,7]$. In these papers, it is assumed that $u \in L^{\infty}\left([0, T] ; W^{1, \infty}(\Omega)\right)$ is a given data and verifies the rather restrictive condition $\operatorname{ess} \inf _{Q}|\nabla u|>0$.

The goal of this work is to state the existence of a weak solution to the whole system $(S)$, such that $\theta, \varphi \in L^{\infty}(Q)$ when the initial data lie in $L^{\infty}(\Omega)$, together with $\theta \geq 0$ and $\varphi \geq 0$ almost everywhere in $Q$ (Theorems 1 and 2). The proofs are based in some different standard techniques, including truncation ( [2]) and a priori estimates. But from the physical (and numerical) standpoint, the results about the $L^{\infty}(Q)$ regularity and positivity of both, $\theta$ and $\varphi$, are very important since, in general, there is an enormous lack of this kind of results in turbulence modelling (e.g. in the $k-\epsilon$ model).

The resolution of a system like $(S)$, or $(C)$ in section 6 , may be regarded, from the mathematical point of view, as a pioneering work in turbulence modelling, since it is the first time that an existence result is shown for a two equations turbulence model.

\section{FUnCtiONAL SPACES AND WEAK FORMULATION}

The following notation and functional spaces will be adopted throughout this paper:

$\Omega \subset \mathbb{R}^{N}$ is a bounded domain with Lispchitz boundary $\partial \Omega ; N \geq 2$ is the space dimension, and $Q$ is the cylinder $\Omega \times(0, T)$, with $T>0$ the final time.

$$
\mathcal{D}(\Omega) \stackrel{\text { def }}{=} \text { space of } C^{\infty} \text { functions with compact support in } \Omega \text {. }
$$

For an integer $m \geq 0$ and $1 \leq p \leq+\infty$ we define

$$
W^{m, p}(\Omega) \stackrel{\text { def }}{=}\left\{v \in L^{p}(\Omega) / \frac{\partial^{|\alpha|} v}{\partial x_{1}^{\alpha_{1}} \cdots \partial x_{N}^{\alpha_{N}}} \in L^{p}(\Omega),\right.
$$




$$
\left.\forall \alpha=\left(\alpha_{1}, \ldots, \alpha_{N}\right) \in \mathbb{Z}_{+}^{N},|\alpha|=\alpha_{1}+\cdots+\alpha_{N} \leq m\right\}
$$

Also, for $s>0$, and $1 \leq p \leq+\infty$ one can introduce the space $W^{s, p}(\Omega)$ by interpolation (see [1]).

$$
\begin{gathered}
W_{0}^{s, p}(\Omega) \stackrel{\text { def }}{=} \text { closure of } \mathcal{D}(\Omega) \text { with the standard norm of } W^{s, p}(\Omega), \\
W^{-s, p^{\prime}}(\Omega) \stackrel{\text { def }}{=} \text { dual space of } W_{0}^{s, p}(\Omega), \quad \frac{1}{p}+\frac{1}{p^{\prime}}=1, \quad 1 \leq p<\infty \\
H^{1}(\Omega) \stackrel{\text { def }}{=} W^{1,2}(\Omega), \quad H_{0}^{1}(\Omega) \stackrel{\text { def }}{=} W_{0}^{1,2}(\Omega), \quad H^{-1}(\Omega) \stackrel{\text { def }}{=} W^{-1,2}(\Omega), \\
\nabla \cdot v \stackrel{\text { def }}{=} \frac{\partial v_{1}}{\partial x_{1}}+\cdots+\frac{\partial v_{N}}{\partial x_{N}}, \quad \text { divergence of } v=\left(v_{1}, \ldots, v_{N}\right)^{\prime}, \\
V \stackrel{\text { def }}{=}\left\{v \in H_{0}^{1}(\Omega)^{N} / \nabla \cdot v=0\right\}, \quad V^{\prime} \stackrel{\text { def }}{=} \text { dual space of } V .
\end{gathered}
$$

Let $n=n(x)$ be the outward unitary normal vector to $\partial \Omega$ in $x \in \partial \Omega$, then we define

$$
H \stackrel{\text { def }}{=}\left\{v \in L^{2}(\Omega)^{N} / \nabla \cdot v=0 \text { in } \Omega, v \cdot n=0 \text { on } \partial \Omega\right\},
$$

For a Banach space $X$ and $1 \leq p \leq+\infty$, we denote by $\boldsymbol{L}^{p}(X)$ the space $L^{p}([0, T] ; X)$, that is, the set of (equivalence class of) functions $f:[0, T] \mapsto X$ measurables and such that $t \in[0, T] \mapsto\|f(t)\|_{X}$ is in $L^{p}(0, T)$. For a function $f \in \boldsymbol{L}^{p}(X)$ we put

$$
\|f\|_{\boldsymbol{L}^{p}(X)} \stackrel{\text { def }}{=}\left(\int_{0}^{T}\|f(t)\|_{X}^{p} d t\right)^{1 / p}, 1 \leq p<+\infty, \quad\|f\|_{\boldsymbol{L}^{\infty}(X)} \stackrel{\text { def }}{=} \underset{t \in[0, T]}{\operatorname{ess} \sup }\|f(t)\|_{X} .
$$

It is well-known that $\left(\boldsymbol{L}^{p}(X),\|\cdot\|_{\boldsymbol{L}^{p}(X)}\right)$ is a Banach space. Notice that by Fubini's theorem we can identify the space $\boldsymbol{L}^{p}\left(L^{p}(\Omega)\right)$ with $L^{p}(Q)$ (the reader is refer to [4] for more properties about these spaces).

$$
\begin{gathered}
\boldsymbol{W}_{1} \stackrel{\text { def }}{=}\left\{v \in \boldsymbol{L}^{2}(V) / \frac{d v}{d t} \in \boldsymbol{L}^{2}\left(V^{\prime}\right)\right\}, \\
\boldsymbol{W}_{2}^{(q)} \stackrel{\text { def }}{=}\left\{v \in \boldsymbol{L}^{2}\left(H_{0}^{1}(\Omega)\right) / \frac{d v}{d t} \in \boldsymbol{L}^{2}\left(W^{-1, q}(\Omega)\right)\right\}, \quad 1 \leq q<+\infty . \\
\boldsymbol{W}_{3}^{(q)} \stackrel{\text { def }}{=}\left\{v \in \boldsymbol{L}^{q}\left(W_{0}^{1, q}(\Omega)\right) / \frac{d v}{d t} \in \boldsymbol{L}^{1}\left(W^{-1, q}(\Omega)\right)\right\}, \quad 1 \leq q<+\infty .
\end{gathered}
$$

In these definitions, all derivatives are assumed to be taken in the sens of distributions. It is well known that all these spaces are Banach spaces provided with their standard norms. Moreover, $V, H$ and $\boldsymbol{W}_{2}^{(2)}$ are in fact 
Hilbert spaces. Also, the following imbeddings hold algebraically and topologically

$$
\left\{\begin{array}{l}
\boldsymbol{W}_{1} \hookrightarrow \boldsymbol{C}(H) ; \quad \boldsymbol{W}_{2}^{(q)} \hookrightarrow \boldsymbol{C}\left(L^{2}(\Omega)\right), \forall q \leq \frac{2 N}{N-2}<\infty ; \\
\boldsymbol{W}_{3}^{(q)} \hookrightarrow \boldsymbol{C}\left(W^{-1, q}(\Omega)\right), \forall q<\infty ;
\end{array}\right.
$$

where we are denoting $\boldsymbol{C}(X)=C([0, T] ; X), X$ a Banach space, the space of continuous functions $v:[0, T] \mapsto X$, provided with the norm $\|v\|_{\boldsymbol{C}(X)}=\max _{[0, T]}\|v(t)\|_{X}$. We will make use of the following compactness lemma (see $[9])$ :

Lemma 1. Let $X, B$ and $Y$ be three Banach spaces such that $X \hookrightarrow B \hookrightarrow Y$, every imbedding being continuous and the inclusion $X \hookrightarrow B$ compact. For $1 \leq p, q<+\infty$, let $\boldsymbol{W}$ be the Banach space defined as $\boldsymbol{W}=$ $\left\{v \in \boldsymbol{L}^{p}(X) / \frac{d v}{d t} \in \boldsymbol{L}^{q}(Y)\right\}$.

Then, the inclusion $\boldsymbol{W} \hookrightarrow \boldsymbol{L}^{p}(B)$ holds and is compact.

Finally, we will use the abbreviation 'a.e.' meaning 'almost everywhere'.

Now, we may introduce the weak formulation of system $(S)$. Let the initial data $u_{0}, \theta_{0}$ and $\varphi_{0}$ (in $\Omega$ ), the boundary constants $a$ and $b$, and the forcing term $f$ (in $Q$ ) be given. Then, we search for $u=\left(u_{1}, \ldots, u_{N}\right)^{\prime}, \theta$ and $\varphi$, in certain suitable spaces, such that:

$$
\begin{gathered}
\int_{0}^{T}\left\langle\frac{d u}{d t}, v\right\rangle+\int_{Q} A(\theta, \varphi) \nabla u \nabla v=\int_{0}^{T}\langle f, v\rangle, \quad \forall v \in L^{2}(V), \\
\int_{0}^{T}\left\langle\frac{d \theta}{d t}, \Psi\right\rangle+\int_{Q} A(\theta, \varphi) \nabla \theta \nabla \Psi=\int_{Q}\left(1-\theta^{2}|\nabla u|^{2}\right) \Psi, \quad \forall \Psi \in \mathcal{D}(Q), \\
\int_{0}^{T}\left\langle\frac{d \varphi}{d t}, \Psi\right\rangle+\int_{Q} A(\theta, \varphi) \nabla \varphi \nabla \Psi=-\int_{Q} \varphi\left(\theta|\nabla u|^{2}+\frac{1}{|\theta|+r}\right) \Psi, \forall \Psi \in \mathcal{D}(Q), \\
u(x, 0)=u_{0}, \quad \theta(x, 0)=\theta_{0}(x), \quad \varphi(x, 0)=\varphi_{0}(x), \quad \text { in } \Omega, \\
\theta(x, t)=a, \quad \varphi(x, t)=b, \quad \text { on } \partial \Omega \times(0, T) .
\end{gathered}
$$

\section{Remarks}

1. In (2)-(4), the symbol $\langle\cdot, \cdot\rangle$ stands for certain duality products specified below.

2. As usual, the pressure $p$ does not appear in the weak formulation (2)-(6) and it can be retrieved by the classical de Rham's argument (see [10]).

3. We will show below that, when $\theta_{0}, \varphi_{0} \in L^{\infty}(\Omega)$ (resp. $\theta_{0}, \varphi_{0} \in L^{1}(\Omega)$ ) then, $\theta-a$ and $\varphi-b$ belong to some space $\boldsymbol{W}_{2}^{(q)}$ (resp. $\boldsymbol{W}_{3}^{(q)}$ ) for some $q \in(1,2)$. In particular, thanks to the imbeddings given in (1), the initial conditions (5) will make sense at least in $L^{2}(\Omega)^{N}$ for $u$, and in $L^{2}(\Omega)$ (resp. $W^{-1, q}(\Omega)$ ) for $\theta$ and $\varphi$.

\section{The MAIn RESUlts}

We will consider the following hypotheses:

(H1) $f \in \boldsymbol{L}^{2}\left(H^{-1}(\Omega)^{N}\right), u_{0} \in H$; 
(H2) $a \geq 0, b \geq 0$ are real constants;

(H3) $\theta_{0} \geq 0, \varphi_{0} \geq 0$;

(H4) $A: \mathbb{R} \times \mathbb{R} \mapsto \mathbb{R}^{N \times N}$ is continuous and there exists a constant $\alpha>0$ such that $A\left(s_{1}, s_{2}\right) \xi \xi \geq \alpha|\xi|^{2}$, for all $s_{1}, s_{2} \in \mathbb{R}, \xi \in \mathbb{R}^{N}$.

The main results now follow. The difference between them is the assumed regularity for the initial data $\theta_{0}$ and $\varphi_{0}$.

Theorem 1. ( $L^{\infty}$ initial data) Under hypotheses $(H 1-H 4)$, if $\theta_{0}, \varphi_{0} \in L^{\infty}(\Omega)$, then there exists $(u, \theta, \varphi)$ solution to (2)-(6), such that

$$
\begin{gathered}
u \in \boldsymbol{W}_{1}, \theta-a, \varphi-b \in \boldsymbol{W}_{2}^{(q)} \cap L^{\infty}(Q), \quad \forall q \in\left[1, \frac{N}{N-1}\right), \\
0 \leq \theta(x, t) \leq \max \left\{\left\|\theta_{0}\right\|_{L^{\infty}(\Omega)}, a\right\}+t, \quad \text { a.e. in } Q, \\
0 \leq \varphi(x, t) \leq \max \left\{\left\|\varphi_{0}\right\|_{L^{\infty}(\Omega)}, b\right\}, \quad \text { a.e. in } Q .
\end{gathered}
$$

Theorem 2. ( $L^{1}$ initial data) Let assume hypotheses $(H 1-H 4)$ and also

(H5) there exists $\beta>0$ such that $\left\|A\left(s_{1}, s_{2}\right)\right\| \leq \beta$ for all $s_{1}, s_{2} \in \mathbb{R},\|\cdot\|$ being some matrix norm.

If $\theta_{0}, \varphi_{0} \in L^{1}(\Omega)$, then there exists $(u, \theta, \varphi)$ solution to (2)-(6), such that

$$
\begin{gathered}
u \in \boldsymbol{W}_{1}, \theta-a, \varphi-b \in \boldsymbol{W}_{3}^{(q)} \cap \boldsymbol{L}^{\infty}\left(L^{1}(\Omega)\right), \quad \forall q \in\left[1, \frac{N+2}{N+1}\right), \\
\theta \geq 0, \quad \varphi \geq 0, \quad \text { a.e. in } Q \\
\theta^{2}|\nabla u|^{2} \in L^{1}(Q), \varphi \theta|\nabla u|^{2} \in L^{1}(Q) .
\end{gathered}
$$

\section{Remarks}

1. In both theorems, due to (1), the initial conditions given in (5) make sens at least in $L^{2}(\Omega)^{N}$ for $u$ and in $W^{-1, q}(\Omega)$ for $\theta$ and $\varphi$.

2. In Section 6 we give two existence results concerning the full system in $2 \mathrm{D}$ with transport terms.

3. We may assume a more general version of the diffusion matrix $A$. Indeed, Theorem 1 also holds if $(H 4)$ is changed to: $A: Q \times \mathbb{R} \times \mathbb{R} \mapsto \mathbb{R}^{N \times N}$ is a Caratheodory matrix function and there exist a constant $\alpha>0$ and a non-decreasing function $d: \mathbb{R}^{+} \mapsto \mathbb{R}^{+}$such that

$$
\alpha|\xi|^{2} \leq A\left(x, t, s_{1}, s_{2}\right) \xi \xi \leq d\left(\left|s_{1}\right|+\left|s_{2}\right|\right)|\xi|^{2}, \quad \text { a.e. in } Q \text {, forall } s_{1}, s_{2} \in \mathbb{R} .
$$

Also, instead of just $A$, we may think of three different viscosities $A_{u}, A_{\theta}$ and $A_{\varphi}$ for the respective equations of $u, \theta$ and $\varphi$. If these three matrix functions verify $\left(H 4^{\prime}\right)$ then Theorem 1 still holds, and if $d$ is constant, then Theorem 2 also holds. 
The next sections are devoted to develop the proofs of Theorems 1 and 2. The basic ideas of the proof are summarized here:

1. To divide the two main difficulties, namely (i) the presence of the terms $\theta^{2}|\nabla u|^{2}$ and $\theta \varphi|\nabla u|^{2}$, which only belong to $L^{1}$; and (ii) the coupling of the $N+2$ equations (2)-(4) through nonlinearities.

2. To avoid $L^{1}$-terms, we use a regularization method based on truncations. This will lead to approximated systems.

3. Finally, the same arguments due to Boccardo and Gallouët [2] may be applied in our context, and this yields the necessary estimates for the approximated solutions to pass to the limit.

\section{Proof of Theorem 1}

\subsection{Setting of the approximated problem $\left(\mathbf{P}_{\mathbf{M}}\right)$}

For every $M>0$, we define the truncation function at height $M, T_{M}$, as

$$
T_{M}(s)=\left\{\begin{array}{ll}
s & \text { if }|s| \leq M, \\
M \operatorname{sign} s & \text { if }|s|>M,
\end{array} \quad \operatorname{sign} s=\left\{\begin{aligned}
s /|s| & \text { if } s \neq 0 \\
0 & \text { if } s=0
\end{aligned}\right.\right.
$$

Then, we consider the approximated problem $\left(P_{M}\right)$ consisting in finding $u_{M}, \theta_{M}$ and $\varphi_{M}$ such that

$\left(P_{M}\right)$

$$
\left\{\begin{array}{l}
u_{M} \in \boldsymbol{W}_{1}, \theta_{M}-a, \varphi_{M}-b \in \boldsymbol{W}_{2}^{(2)} \cap L^{\infty}(Q) \text { and, } \\
\int_{0}^{T}\left\langle\frac{d u_{M}}{d t}, v\right\rangle+\int_{Q} A\left(\theta_{M}, \varphi_{M}\right) \nabla u_{M} \nabla v=\int_{0}^{T}\langle f, v\rangle, \quad \forall v \in \boldsymbol{L}^{2}(V), \\
\int_{0}^{T}\left\langle\frac{d \theta_{M}}{d t}, \Psi\right\rangle+\int_{Q} A\left(\theta_{M}, \varphi_{M}\right) \nabla \theta_{M} \nabla \Psi \\
=\int_{Q}\left(1-\theta_{M}\left|\theta_{M}\right| T_{M}\left(\left|\nabla u_{M}\right|^{2}\right)\right) \Psi, \forall \Psi \in \boldsymbol{L}^{2}\left(H_{0}^{1}(\Omega)\right), \\
\int_{0}^{T}\left\langle\frac{d \varphi_{M}}{d t}, \Psi\right\rangle+\int_{Q} A\left(\theta_{M}, \varphi_{M}\right) \nabla \varphi_{M} \nabla \Psi \\
=-\int_{Q} \varphi_{M}\left(\left|\theta_{M}\right| T_{M}\left(\left|\nabla u_{M}\right|^{2}\right)+\frac{1}{\left|\theta_{M}\right|+r}\right) \Psi, \forall \Psi \in \boldsymbol{L}^{2}\left(H_{0}^{1}(\Omega)\right), \\
u_{M}(0)=u_{0}, \quad \theta_{M}(0)=\theta_{0}, \quad \varphi_{M}(0)=\varphi_{0} .
\end{array}\right.
$$

The existence of solution to $\left(P_{M}\right)$ is guaranteed by the next

Lemma 2. Under hypotheses $(H 1-H 4)$, there exists a solution $\left(u_{M}, \theta_{M}, \varphi_{M}\right)$ of problem $\left(P_{M}\right)$ such that

$$
\begin{aligned}
& 0 \leq \theta_{M}(x, t) \leq \max \left\{a,\left\|\theta_{0}\right\|_{L^{\infty}(\Omega)}\right\}+t, \quad \text { a.e. in } \Omega, \forall t \in[0, T] \\
& 0 \leq \varphi_{M}(x, t) \leq \max \left\{b,\left\|\varphi_{0}\right\|_{L^{\infty}(\Omega)}\right\}, \quad \text { a.e. in } \Omega, \forall t \in[0, T] .
\end{aligned}
$$

The existence of $\left(u_{M}, \theta_{M}, \varphi_{M}\right)$ solution to $\left(P_{M}\right)$ and verifying the $L^{\infty}$ estimates (14)-(15) can be found in [5]. Basically, it is obtained by an application of Schauder's fix point theorem.

\subsection{Estimates for $\left(\mathbf{u}_{\mathrm{M}}, \boldsymbol{\theta}_{\mathrm{M}}, \varphi_{\mathrm{M}}\right)$}

Estimates for $\left(u_{M}\right)$

The classical estimates for the Stokes (or Navier-Stokes) equations follows inmediately by taking $v=$ $u_{M}$ as a test function in the equation verified by $u_{M}$ in $\left(P_{M}\right)$. This leads to the existence of a constant 
$C_{1}=C_{1}\left(\alpha,\|f\|_{L^{2}\left(H^{-1}(\Omega)\right.}, T,\left\|u_{0}\right\|_{L^{2}(\Omega)}\right)$ such that

$$
\left\|u_{M}\right\|_{\boldsymbol{L}^{\infty}\left(L^{2}(\Omega)^{N}\right)} \leq C_{1}, \quad\left\|u_{M}\right\|_{\boldsymbol{L}^{2}(V)} \leq C_{1} .
$$

Estimates for $\theta_{M}$ and $\varphi_{M}$

First of all, Lemma 2 gives uniform $L^{\infty}$ bounds for $\left(\theta_{M}\right)$ and $\left(\varphi_{M}\right)$. Now, taking $\phi=\theta_{M}-a$ and $\phi=\varphi_{M}-b$ in the respective equations for $\theta_{M}$ and $\varphi_{M}$, yield

$$
\left\|\theta_{M}\right\|_{L^{2}\left(H^{1}(\Omega)\right)} \leq C_{2}, \quad\left\|\varphi_{M}\right\|_{L^{2}\left(H^{1}(\Omega)\right)} \leq C_{3}
$$

where $C_{2}=C_{2}\left(C_{1}, a,\left\|\theta_{0}\right\|_{L^{\infty}(\Omega)}\right)$ and $C_{3}=C_{3}\left(C_{2}, b,\left\|\varphi_{0}\right\|_{L^{\infty}(\Omega)}\right)$.

Time derivatives estimates

From the estimates derived in the preceeding paragraphs, we may deduce that $\left(\frac{d u_{M}}{d t}\right)$ is bounded in $\boldsymbol{L}^{2}\left(H^{-1}(\Omega)^{N}\right)$, whereas $\left(\frac{d \theta_{M}}{d t}\right)$ and $\left(\frac{d \varphi_{M}}{d t}\right)$ are bounded in $\boldsymbol{L}^{2}\left(H^{-1}(\Omega)\right)+\boldsymbol{L}^{2}\left(L^{1}(\Omega)\right)$. On the other hand, we know by Sobolev's imbedding (see $[3])$ that $L^{1}(\Omega) \hookrightarrow W^{-1, q}(\Omega)$ whenever $q<\frac{N}{N-1}$, which implies the inclusion $\boldsymbol{L}^{2}\left(L^{1}(\Omega)\right) \hookrightarrow \boldsymbol{L}^{2}\left(W^{-1, q}(\Omega)\right)$. Finally,

$$
\boldsymbol{L}^{2}\left(H^{-1}(\Omega)\right)+\boldsymbol{L}^{2}\left(L^{1}(\Omega)\right) \hookrightarrow \boldsymbol{L}^{2}\left(W^{-1, q}(\Omega)\right), \quad \forall q<\frac{N}{N-1}
$$

and we may conclude that

$$
\left(\frac{d \theta_{M}}{d t}\right), \quad\left(\frac{d \varphi_{M}}{d t}\right) \quad \text { are bounded in } \boldsymbol{L}^{2}\left(W^{-1, q}(\Omega)\right), \forall q<\frac{N}{N-1} .
$$

Now, the inclusions $X=H^{1}(\Omega) \hookrightarrow B=L^{2}(\Omega) \hookrightarrow Y=W^{-1, q}(\Omega)$ verify the hypotheses of Lemma 1 , and consequently the imbedding $\boldsymbol{W}=\boldsymbol{W}_{2}^{(q)} \hookrightarrow L^{2}(Q)$ is compact. In particular, this implies that $\left(\theta_{M}\right)$ and $\left(\varphi_{M}\right)$ are relative compact in $L^{2}(Q)$.

\subsection{Passing to the limit in $\left(\mathbf{P}_{\mathbf{M}}\right)$}

From the estimates obtained in the last section, we deduce that from $\left(u_{M}\right),\left(\theta_{M}\right)$ and $\left(\varphi_{M}\right)$ we may extract subsequences, denoted in the same way, such that

$$
\begin{gathered}
u_{M} \rightarrow u \in \boldsymbol{W}_{1}\left\{\begin{array}{l}
\text { in } \boldsymbol{L}^{2}\left(H_{0}^{1}(\Omega)^{N}\right) \text {-weakly, } \\
\text { in } L^{2}(Q)^{N} \text {-strongly, } \\
\text { a.e. in } Q, \\
\text { in } \boldsymbol{L}^{\infty}\left(L^{2}(\Omega)^{N}\right) \text {-weak-*, }
\end{array}\right. \\
\begin{aligned}
\theta_{M} \rightarrow \theta, \quad \varphi_{M} \rightarrow \varphi, \\
\theta-b \in \boldsymbol{W}_{2}^{(q)}, \forall q<\frac{N}{N-1},
\end{aligned}\left\{\begin{array}{l}
\text { in } \boldsymbol{L}^{2}\left(H^{1}(\Omega)\right) \text {-weakly, } \\
\text { in } L^{2}(Q) \text {-strongly, } \\
\text { a.e. in } Q, \\
\text { in } L^{\infty}(Q) \text {-weak-* },
\end{array}\right. \\
\frac{d u_{M}}{d t} \rightarrow \frac{d u}{d t}, \quad \text { in } \boldsymbol{L}^{2}\left(H^{-1}(\Omega)\right) \text {-weakly }
\end{gathered}
$$




$$
\frac{d \theta_{M}}{d t} \rightarrow \frac{d \theta}{d t}, \quad \frac{d \varphi_{M}}{d t} \rightarrow \frac{d \varphi}{d t}, \quad \text { in } \boldsymbol{L}^{2}\left(W^{-1, q}(\Omega)\right) \text {-weakly, } \quad \forall q<\frac{N}{N-1} .
$$

These convergences are enough to pass to the limit in the velocity equation of $\left(P_{M}\right)$, obtaining then (2). But passing to the limit in the $\theta_{M}$ and $\varphi_{M}$ equations cannot be done directly unless the convergence of $\left(u_{M}\right)$ to $u$ is in $\boldsymbol{L}^{2}\left(H_{0}^{1}(\Omega)^{N}\right)$-strongly. Fortunately, this is the case as it can be shown by taking $v=u_{M}$ in $\left(P_{M}\right)$ and passing to the limit.

Consequently, $\left|\nabla u_{M}\right|^{2} \rightarrow|\nabla u|^{2}$ in $L^{1}(Q)$-strongly and, without loss of generality, we may assume that the convergence also holds almost eveywhere in $Q$. Now, we may pass to the limit in the $\theta_{M}$ and $\varphi_{M}$ equations and deduce (3) and (4), respectively.

On the other hand, using (1), we readily obtain (5). This ends the proof of Theorem 1.

\section{Proof of Theorem 2}

Now, we assume hypothesis $(H 5)$ and that $\theta_{0}, \varphi_{0} \in L^{1}(\Omega)$. For $M>0$, we consider the new approximated problem $\left(Q_{M}\right)$

$\left(Q_{M}\right)$

$$
\left\{\begin{array}{l}
u_{M} \in \boldsymbol{W}_{1}, \theta_{M}-a, \varphi_{M}-b \in \boldsymbol{W}_{2}^{(2)} \cap L^{\infty}(Q) \text { and such that } \\
\int_{0}^{T}\left\langle\frac{d u_{M}}{d t}, v\right\rangle+\int_{Q} A\left(\theta_{M}, \varphi_{M}\right) \nabla u_{M} \nabla v=\int_{0}^{T}\langle f, v\rangle, \quad \forall v \in \boldsymbol{L}^{2}(V), \\
\int_{0}^{T}\left\langle\frac{d \theta_{M}}{d t}, \Psi\right\rangle+\int_{Q} A\left(\theta_{M}, \varphi_{M}\right) \nabla \theta_{M} \nabla \Psi \\
\quad=\int_{Q}\left(1-\theta_{M}^{2} T_{M}\left(\left|\nabla u_{M}\right|^{2}\right)\right) \Psi, \forall \Psi \in \boldsymbol{L}^{2}\left(H_{0}^{1}(\Omega)\right), \\
\int_{0}^{T}\left\langle\frac{d \varphi_{M}}{d t}, \Psi\right\rangle+\int_{Q} A\left(\theta_{M}, \varphi_{M}\right) \nabla \varphi_{M} \nabla \Psi \\
\quad=-\int_{Q} \varphi_{M}\left(\theta_{M} T_{M}\left(\left|\nabla u_{M}\right|^{2}\right)+\frac{1}{\theta_{M}+r}\right) \Psi, \forall \Psi \in \boldsymbol{L}^{2}\left(H_{0}^{1}(\Omega)\right), \\
u_{M}(0)=u_{0}, \quad \theta_{M}(0)=T_{M}\left(\theta_{0}\right), \quad \varphi_{M}(0)=T_{M}\left(\varphi_{0}\right) .
\end{array}\right.
$$

Since $T_{M}\left(\theta_{0}\right), T_{M}\left(\varphi_{0}\right) \in L^{\infty}(\Omega)$ we can apply Lemma 2 and deduce that $\left(Q_{M}\right)$ admits a solution $\left(u_{M}, \theta_{M}, \varphi_{M}\right)$.

It is straightforward that the estimates for $\left(u_{M}\right)$ given in (16) still hold. For $\left(\theta_{M}\right)$ and $\left(\varphi_{M}\right)$ we have the

Lemma 3. Let $\tilde{\theta}_{M}=\theta_{M}-a$ and $\tilde{\varphi}_{M}=\varphi_{M}-b$. Then, there exists a constant $C>0$ (depending on $\left.\left(\|f\|_{L^{2}\left(H^{-1}\right)}, \alpha, a, b, T,\left\|\theta_{0}\right\|_{L^{1}(\Omega)},\left\|\varphi_{0}\right\|_{L^{1}(\Omega)}\right)\right)$ such that for all $M>0$

$$
\begin{gathered}
\int_{\Omega}\left|\tilde{\theta}_{M}(t)\right| \leq C, \forall t \in[0, T], \quad \int_{Q} \theta_{M}^{2} T_{M}\left(\left|\nabla u_{M}\right|^{2}\right) \leq C ; \\
\int_{\Omega}\left|\tilde{\varphi}_{M}(t)\right| \leq C, \forall t \in[0, T], \quad \int_{Q} \varphi_{M} \theta_{M} T_{M}\left(\left|\nabla u_{M}\right|^{2}\right) \leq C ; \\
\left\|\nabla T_{j}\left(\tilde{\theta}_{M}\right)\right\|_{\boldsymbol{L}^{2}\left(H_{0}^{1}(\Omega)\right)} \leq C, \quad\left\|\nabla T_{j}\left(\tilde{\varphi}_{M}\right)\right\|_{\boldsymbol{L}^{2}\left(H_{0}^{1}(\Omega)\right)} \leq C, \quad \forall j>0 ;
\end{gathered}
$$




$$
\int_{B_{M}^{j}} A\left(\theta_{M}, \varphi_{M}\right) \nabla \tilde{\theta}_{M} \nabla \tilde{\theta}_{M} \leq C, \quad \int_{C_{M}^{j}} A\left(\theta_{M}, \varphi_{M}\right) \nabla \tilde{\varphi}_{M} \nabla \tilde{\varphi}_{M} \leq C, \quad \forall j>0,
$$

where $B_{M}^{j}=\left\{(x, t) \in Q / j \leq\left|\tilde{\theta}_{M}\right|<j+1\right\}$ and $C_{M}^{j}=\left\{(x, t) \in Q / j \leq\left|\tilde{\varphi}_{M}\right|<j+1\right\}$;

$$
\lim _{j \rightarrow \infty} \int_{\left\{\theta_{M}>j\right\}} \theta_{M}^{2} T_{M}\left(\left|\nabla u_{M}\right|^{2}\right)=\lim _{j \rightarrow \infty} \int_{\left\{\varphi_{M}>j\right\}} \varphi_{M} \theta_{M} T_{M}\left(\left|\nabla u_{M}\right|^{2}\right)=0 .
$$

Proof. All the estimates are obtained by using in $\left(Q_{M}\right)$ suitable test functions. Indeed, (18) and (19) are deduced taking $\phi=\frac{1}{\varepsilon} T_{\varepsilon}\left(\tilde{\theta}_{M}\right)$ and $\phi=\frac{1}{\varepsilon} T_{\varepsilon}\left(\tilde{\varphi}_{M}\right)$ in the $\theta_{M}$ and $\varphi_{M}$ equations respectively, and then passing to the limit in $\varepsilon \downarrow 0$.

Estimates in (20) are straightforward by putting $\phi=T_{j}\left(\tilde{\theta}_{M}\right)$ (resp. $\phi=T_{j}\left(\tilde{\varphi}_{M}\right)$ ). Finally, to obtain (21) and (22) we just take $\phi=g_{j}\left(\tilde{\theta}_{M}\right)\left(\right.$ resp. $\left.\phi=g_{j}\left(\tilde{\varphi}_{M}\right)\right)$ where $g_{j}$ is given by

$$
g_{j}(s)= \begin{cases}0 & \text { if }|s|<j \\ \operatorname{sign} s & \text { if }|s| \geq j+1 \\ s-j \operatorname{sign} s & \text { if } j \leq|s|<j+1 .\end{cases}
$$

Now, we may apply a result due to Boccardo and Gallouët [2] and deduce, from $(20,21)$ that $\left(\theta_{M}\right)$ and $\left(\varphi_{M}\right)$ are bounded in $\boldsymbol{L}^{q}\left(W^{1, q}(\Omega)\right)$, for all $q<\frac{N+2}{N+1}$. Going back to $\left(Q_{M}\right)$, we see that $\left(\frac{d u_{M}}{d t}\right)$ is bounded in $\boldsymbol{L}^{2}\left(H^{-1}(\Omega)^{N}\right)$, whereas $\left(\frac{d \theta_{M}}{d t}\right)$ and $\left(\frac{d \varphi_{M}}{d t}\right)$ are bounded in $L^{1}(Q)+\boldsymbol{L}^{q}\left(W^{-1, q}(\Omega)\right)$, for all $q<\frac{N+2}{N+1}$ (here, we have explicitly used hypothesis $(H 5))$. But now, we have $L^{1}(Q) \hookrightarrow \boldsymbol{L}^{1}\left(W^{-1, r}(\Omega)\right)$ whenever $r<\frac{N}{N-1}$, and since $\frac{N+2}{N+1}<\frac{N}{N-1}$, $N \geq 2$, we also have

$$
L^{1}(Q)+\boldsymbol{L}^{q}\left(W^{-1, q}(\Omega)\right) \hookrightarrow \boldsymbol{L}^{1}\left(W^{-1, q}(\Omega)\right), \quad \forall q<\frac{N+2}{N+1} .
$$

This means that $\left(\tilde{\theta}_{M}\right)$ and $\left(\tilde{\varphi}_{M}\right)$ lie in a bounded set of the space $\boldsymbol{W}_{3}^{(q)}, \forall q<\frac{N+2}{N+1}$, which is compactly imbedded in $\boldsymbol{L}^{1}\left(L^{q}(\Omega)\right)$ thanks to Lemma 1.

\subsection{Passing to the limit in $\left(\mathbf{Q}_{\mathbf{M}}\right)$}

From the estimates obtained above, we deduce that from $\left(u_{M}\right),\left(\theta_{M}\right)$ and $\left(\varphi_{M}\right)$ we may extract subsequences, denoted in the same way, such that

$$
\begin{gathered}
u_{M} \rightarrow u \in \boldsymbol{W}_{1}\left\{\begin{array}{l}
\text { in } \boldsymbol{L}^{2}\left(H_{0}^{1}(\Omega)^{N}\right) \text {-weakly, } \\
\text { in } L^{2}(Q)^{N} \text {-strongly, } \\
\text { a.e. in } Q, \\
\text { in } \boldsymbol{L}^{\infty}\left(L^{2}(\Omega)^{N}\right) \text {-weak-*, }
\end{array}\right. \\
\theta-a, \varphi-b \in \boldsymbol{W}_{3}^{(q)}, \forall q<\frac{N+2}{N+1},\left\{\begin{array}{l}
\text { in } \boldsymbol{L}^{q}\left(W^{1, q}(\Omega)\right) \text {-weakly, } \\
\text { in } \boldsymbol{L}^{1}\left(L^{q}(\Omega)\right) \text {-strongly } \\
\text { a.e. in } Q,
\end{array}\right.
\end{gathered}
$$




$$
\begin{gathered}
\frac{d u_{M}}{d t} \rightarrow \frac{d u}{d t}, \quad \text { in } \boldsymbol{L}^{2}\left(H^{-1}(\Omega)\right) \text {-weakly } \\
\frac{d \theta_{M}}{d t} \rightarrow \frac{d \theta}{d t}, \quad \frac{d \varphi_{M}}{d t} \rightarrow \frac{d \varphi}{d t}, \quad \text { in } \mathcal{D}^{\prime}(Q) .
\end{gathered}
$$

\section{Remark}

Notice that from the respectively convergences of $\left(\frac{d \theta_{M}}{d t}\right)$ and $\left(\frac{d \phi_{M}}{d t}\right)$, we just obtain $\frac{d \theta}{d t}, \frac{d \phi}{d t} \in \mathcal{D}^{\prime}(Q)$. The conclusion $\frac{d \theta}{d t}, \frac{d \phi}{d t} \in \boldsymbol{L}^{1}\left(W^{-1, q}(\Omega)\right)$ is derived a posteriori, that is, from the equations verified by $\theta$ and $\varphi$, respectively.

As in $\left(P_{M}\right)$, we can show that the convergence $u_{M} \rightarrow u$ still holds $\boldsymbol{L}^{2}\left(H_{0}^{1}(\Omega)\right)$-strongly and we may assume that

$$
\left|\nabla u_{M}\right|^{2} \rightarrow|\nabla u|^{2}, \quad \text { a.e. in } Q
$$

By (18), (19), (23) and Fatou's lemma, we derive (12). Then, using this fact and (22) and (23), we obtain

$$
\theta_{M}^{2} T_{M}\left(\left|\nabla u_{M}\right|^{2}\right) \rightarrow \theta^{2}|\nabla u|^{2}, \quad \varphi_{M} \theta_{M} T_{M}\left(\left|\nabla u_{M}\right|^{2}\right) \rightarrow \varphi \theta|\nabla u|^{2}, \quad \text { in } L^{1}(Q) .
$$

Consequently, all terms in $\left(Q_{M}\right)$ pass to the limit. It remains to prove that $\theta, \varphi \in \boldsymbol{L}^{\infty}\left(L^{1}(\Omega)\right)$; but this is a straightforward consequence of Fatou's lemma.

This ends the proof of Theorem 2.

\section{Concluding REMARKS}

Notice that Theorems 1 and 2 hold for all $N \geq 2$, the space dimension, though it intervenes in the regularity of the solutions $\theta$ and $\varphi$.

In order to study the full system, including transport terms, namely

$$
\begin{cases}\frac{\partial u}{\partial t}+(u \cdot \nabla) u-\nabla \cdot(A(\theta, \varphi) \nabla u)+\nabla p=f, & \nabla \cdot u=0, \\ \frac{\partial \theta}{\partial t}+u \nabla \theta-\nabla \cdot(A(\theta, \varphi) \nabla \theta)=1-\theta^{2}|\nabla u|^{2}, & \text { in } Q \\ \frac{\partial \varphi}{\partial t}+u \nabla \varphi-\nabla \cdot(A(\theta, \varphi) \nabla \varphi)=-\varphi\left(\theta|\nabla u|^{2}+\frac{1}{\theta+r}\right), & \text { in } Q \\ u(x, 0)=u_{0}(x), \quad \theta(x, 0)=\theta_{0}(x), \quad \varphi(x, 0)=\varphi_{0}(x), & \text { in } \Omega \\ u(x, t)=0, \quad \theta(x, t)=a, \quad \varphi(x, t)=b, & \text { on } \partial \Omega \times(0, T)\end{cases}
$$

we ought to restrict ourselves to study the case $N=2$, since the process described in this work fails because it is not known if the energy identity is still verified (i.e. in $N=3$, the convergence $\left|\nabla u_{M}\right|^{2} \rightarrow|\nabla u|^{2}$ in $L^{1}(Q)$-strongly is not guaranted). 
By a weak solution of system $(C)$ we mean a triplet $(u, \theta, \varphi)$, such that (remember that the pressure may be retrieved by the usual de Rham's argument)

$$
\left\{\begin{array}{l}
u \in \boldsymbol{W}_{1}, \quad \theta-a, \varphi-b \in \boldsymbol{W}_{3}^{(q)}, \theta^{2}|\nabla u|^{2}, \varphi \theta|\nabla u|^{2} \in L^{1}(Q), \\
\int_{0}^{T}\left\langle\frac{d u}{d t}, v\right\rangle+\int_{Q}(u \cdot \nabla) u v+\int_{Q} A(\theta, \varphi) \nabla u \nabla v=\int_{0}^{T}\langle f, v\rangle, \quad \forall v \in \boldsymbol{L}^{2}(V), \\
\int_{0}^{T}\left\langle\frac{d \theta}{d t}, \Psi\right\rangle-\int_{Q} u \nabla \Psi \theta+\int_{Q} A(\theta, \varphi) \nabla \theta \nabla \Psi=\int_{Q}\left(1-\theta^{2}|\nabla u|^{2}\right) \Psi \\
\forall \Psi \in \mathcal{D}(Q), \\
\int_{0}^{T}\left\langle\frac{d \varphi}{d t}, \Psi\right\rangle-\int_{Q} u \nabla \Psi \varphi+\int_{Q} A(\theta, \varphi) \nabla \varphi \nabla \Psi \\
=\int_{Q}-\varphi\left(\theta|\nabla u|^{2}+\frac{1}{\theta+r}\right) \Psi, \quad \forall \Psi \in \mathcal{D}(Q) \\
u(0)=u_{0}, \quad \theta(0)=\theta_{0}, \quad \varphi(0)=\varphi_{0}, \quad \text { in } \Omega
\end{array}\right.
$$

Then, the following theorem holds

Theorem 3. Let $N=2$ and assume hypotheses $(H 1-H 4)$.

(i) If $\theta_{0}, \varphi_{0} \in L^{\infty}(\Omega)$, then there exists a solution to $(D)$ such that (7)-(9) are verified.

(ii) If $\theta_{0}, \varphi_{0} \in L^{1}(\Omega)$ and (H5) is assumed, then there exists a solution to $(D)$ such that (10)-(12) are verified.

Proof. We apply the same technique described above and we just need to show that all transport terms pass to the limit. To this end, we know that, when $N=2, u_{M} \in L^{4}(Q)$ and $u_{M} \rightarrow u$ strongly in this space; now, in the first case, we have $u_{M} \theta_{M} \rightarrow u \theta, u_{M} \varphi_{M} \rightarrow u \varphi$ in $L^{4}(Q)$-strongly, and then $\nabla \cdot\left(u_{M} \theta_{M}\right) \rightarrow \nabla \cdot(u \theta)$, $\nabla \cdot\left(u_{M} \varphi_{M}\right) \rightarrow \nabla \cdot(u \varphi)$ in $\boldsymbol{L}^{4}\left(W^{-1,4}(\Omega)\right)$-strongly.

In the second case, the interpolation between $\boldsymbol{L}^{q}\left(W^{1, q}\right)(q<4 / 3)$ and $\boldsymbol{L}^{\infty}\left(L^{1}(\Omega)\right)$ yields $\left(\theta_{M}\right)$ and $\left(\varphi_{M}\right)$ bounded in $L^{r}(Q)$, for all $r<2$, so that $u_{M} \theta_{M} \rightarrow u \theta, u_{M} \varphi_{M} \rightarrow u \varphi$ in $L^{r}(Q)$-weakly. Hence, $\nabla \cdot\left(u_{M} \theta_{M}\right) \rightarrow$ $\nabla \cdot(u \theta), \nabla \cdot\left(u_{M} \varphi_{M}\right) \rightarrow \nabla \cdot(u \varphi)$ in $\boldsymbol{L}^{q}\left(W^{-1, q}(\Omega)\right)$-weakly, for all $q<4 / 3$.

There is no uniqueness result for this kind of problems up till now.

The authors wish to thank Dr. E. Fernández Cara, Dr. R. Lewandowski and Dr. F. Murat for fruitful discussions and useful comments and suggestions.

\section{REFERENCES}

[1] R. Adams, Sobolev Spaces. Academic Press (1975).

[2] L. Boccardo and T. Gallouët, Non-linear Elliptic and Parabolic Equations Involving Meausure Data. J. Funct. Anal. 87 (1989) 149-169.

[3] H. Brezis, Analyse Fonctionnelle. Théorie et Applications. Masson, Paris (1983).

[4] T. Cazenave and A. Haraux, Introduction aux problèmes d'évolution semi-linéaires. Série Mathématiques et Applications, Ellipses (1990).

[5] M. Gómez Mármol, Ph.D. thesis. Universidad de Sevilla (to appear).

[6] R. Lewandowski, Modèles de turbulence et équations paraboliques. C. R. Acad. Sci. Paris 317 (1993) 835-840.

[7] R. Lewandowski and B. Mohammadi, Existence and positivity results for the $\phi-\theta$ model and a modified $k-\epsilon$ turbulence model. Math. Model and Methods in Applied Sciences 3 (1993) 195-215.

[8] B. Mohammadi and O. Pironneau, Analysis of the $k-\epsilon$ turbulence model. Research in Applied Mathematics. Wiley-Masson, Paris (1994).

[9] J. Simon, Compact sets in the space $L^{p}([0, T] ; B)$. Annali di Matemàtica Pura et Applicata (1987) 65-96.

[10] Temam, R. Navier-Stokes equations. Theory and numerical analysis. North Holland Publishing Company, Amsterdam (1979). 